Article

\title{
'Dose-to-Mother' Deuterium Oxide Dilution Technique: An Accurate Strategy to Measure Vitamin A Intake in Breastfed Infants
}

\author{
Veronica Lopez-Teros ${ }^{1, *}$, Ana Teresa Limon-Miro ${ }^{2}$, Humberto Astiazaran-Garcia ${ }^{2}$, \\ Sherry A. Tanumihardjo ${ }^{3}$, Orlando Tortoledo-Ortiz ${ }^{2}$ and Mauro E. Valencia ${ }^{1}$ \\ 1 Nutritional Sciences, Department of Chemical and Biological Sciences, University of Sonora, Hermosillo, \\ Sonora 83000, Mexico; mauro@ciad.mx \\ 2 Department of Nutrition, Research Center for Food and Development (CIAD, AC), Hermosillo, \\ Sonora 83304, Mexico; analimonmiro@gmail.com (A.T.L.-M.); hastiazaran@ciad.mx (H.A.-G.); \\ otortoledo@ciad.mx (O.T.-O.) \\ 3 Department of Nutritional Sciences, University of Wisconsin-Madison, Madison, WI 53706, USA; \\ sherry@nutrisci.wisc.edu \\ * Correspondence: veronica.lopez@unison.mx; Tel.: +52-662-289-3781
}

Received: 31 October 2016; Accepted: 16 February 2017; Published: 21 February 2017

\begin{abstract}
In Mexico, infants (0-2 years old) show the highest prevalence of vitamin A deficiency (VAD), measured by serum retinol concentrations. Thus, we consider that low vitamin A (VA) intake through breast milk (BM) combined with poor weaning practices are the main factors that contribute to VAD in this group. We combined the assessment of VA status in lactating women using BM retinol and a stable isotope 'dose-to-mother' technique to measure BM production in women from urban and agricultural areas. Infants' mean BM intake was $758 \pm 185 \mathrm{~mL}$, and no difference was observed between both areas ( $p=0.067)$. Mean BM retinol concentration was $1.09 \mu \mathrm{mol} / \mathrm{L}$, which was significantly lower for the agricultural area $(p=0.028)$. Based on BM retinol concentration, $57 \%$ of women were VAD; although this prevalence fell to $16 \%$ when based on fat content. Regardless of the VA biomarker used here, infants from the urban and agricultural areas cover only $66 \%$ and $49 \%$ of their dietary adequate intake from BM, respectively $(p=0.054)$. Our data indicate that VAD is still a public health concern in Mexico. Adopting both methods to assess VA transfer from the mother to the breastfed child offers an innovative approach towards the nutritional assessment of vulnerable groups.
\end{abstract}

Keywords: deuterium oxide dilution; infants' vitamin A adequacy; breast milk retinol; breast milk carotenoids; breast milk intake; VA status in lactating women

\section{Introduction}

Vitamin A deficiency (VAD) is recognized by the World Health Organization (WHO) as one of the main nutritional problems that affects developing countries, where children under 5 years, and pregnant and lactating women are the most vulnerable groups [1]. In Mexico, VAD is a severe subclinical public health problem, particularly for children $<5$ years old; unfortunately, there is not much information on the VA status of Mexican pregnant and lactating women.

A study performed in lactating women from northwest Mexico (Sonora) [2], showed that more than $50 \%$ had VAD using serum retinol concentrations as an indicator, which is indicative of a severe public health problem [1]. Additionally, the mean breast milk (BM) retinol concentration was $1.18 \mu \mathrm{mol} / \mathrm{L}$, which is close to $1 \mu \mathrm{mol} / \mathrm{L}$ observed in regions where VAD is common [3]. This VA concentration in breast milk is barely enough to satisfy the metabolic needs of the breastfed child, leaving little opportunity to accumulate VA reserves in the liver for times of low intake [4]. 
The Mexican National Nutrition Survey 2012 [5], shows that VAD is of concern in children $<2$ years old. This suggests that low intake of VA through BM combined with poor weaning practices could be the main factors contributing to VAD in this age group.

Efforts to reduce VAD in children under 5 years have been carried out for over 20 years in Mexico. Preformed VA supplements are administered to children 6 months to 4 years old $(60 \mathrm{mg}$; $210 \mu \mathrm{mol}$ retinyl palmitate) during national immunization campaigns. Although there is an immediate improvement in VA status, its effect is limited in time ( $\sim 3$ months), whereby circulating VA returns to pre-dose concentrations and VAD reappears in areas with low VA intakes [6]. Additionally, recent estimates in a sample of preschoolers from northwest Mexico, showed that $48 \%$ had low serum retinol concentrations [7].

Given the above, to adequately fight VAD in breastfed infants, it is essential that the mother's VA status be optimal in order to fulfill the infant's metabolic needs and be sufficient to maintain VA stores and prevent VAD [4]. In lactating women, $\mathrm{BM}$ retinol concentration is a unique indicator related to the mother's VA status, is sensitive to dietary VA intake [8,9], and may predict the breastfed infant's VA status [10,11]. Furthermore, BM retinol may not be affected by systemic inflammation [12], although this requires more research considering that serum retinol bound to retinol-binding protein contributes to BM retinol [13] and is decreased during inflammation [14].

Even though BM has a central role in energy and nutrient intake, which are essential for the infant's growth and development, only limited records document BM intake [15]. Furthermore, the only available information associating BM intake and VAD was performed in Senegalese breastfed infants showing that VA intake was close to their recommended dietary adequate intake [16].

The gold standard to measure BM intake in humans is the 'Dose-to-mother' deuterium oxide dilution (DOD) technique $[17,18]$, which measures the turnover rate of body water after an oral dose of deuterium oxide (stable isotope-tracer) provided to the lactating mother. Thus, through BM, the tracer is transferred from mother to child, allowing the quantification of BM volume consumed by the infant. Additionally, the method discriminates between exclusive and partial breastfeeding and does not require strict schedules for BM sampling, allowing a more natural study of BM intake without interfering with the normal activities of the mother-infant pair.

Considering the above, in order to reduce VAD in nursing infants, we need a further understanding of the association between the VA status of the mother and its impact on the breastfed infant. Therefore, we aimed to combine two methods (i.e., dose-to-mother DOD and BM retinol concentration based on volume and fat content), to assess the VA status of lactating women, estimate the transfer of retinol from the mother to the breastfed child, and evaluate the nutritional adequacy of VA in breastfed children from an urban and an agricultural area.

\section{Subjects and Methods}

\subsection{Study Design and Bioethical Considerations}

This was a cross-sectional design where breastfed infants and their mothers were selected by invitation from one of two study areas, i.e., urban and agricultural areas, in Sonora, Mexico. The study was approved by the Bioethics and Research Committee from the Department of Medicine and Health Sciences of University of Sonora (DCMS/CBIDMCS/D-23 20 March 2012; addendum for BM retinol analysis: DMCS/CBIDMCS/D-37 12 February 2013) endorsed by the Mexican National Bioethics Commission. Written informed consent was obtained from participants.

The study areas included Hermosillo, the capital city of Sonora state, as the urban area and Poblado Miguel Aleman, located in the Sonoran Coast, as the agricultural area. Participants who declared exclusive breastfeeding (EBF) or predominant breastfeeding (PBF) were recruited from local health clinics participating in health counseling programs during October 2012-March 2013. 
Sample size was calculated based on a theoretical BM volume difference of $100 \mathrm{~mL}$ between EBF and PBF women and a standard deviation of $130 \mathrm{~mL} /$ day with an alpha level of 0.05 and a beta of 0.80 [18]. Thus, the calculated sample size was 30 mother-infant pairs from each area.

\subsection{Selection Criteria}

Adult mothers who declared EBF or PBF practices and resided for more than a year in one of the two study areas, were eligible to participate in the study. Additionally, their infants had to be born at full-term pregnancy (38-42 weeks gestation) and 3-6 months old at recruitment. Exclusion criteria included multiple births or infants in the weaning process, as well as mothers that consumed alcohol, tobacco, or drugs. Mothers who failed to complete the 'dose-to-mother' protocol and infants whose quantified BM intake was below $250 \mathrm{~mL} /$ day, were excluded because in a well-established breastfeeding process the infant's BM intake is typically between 620 and $660 \mathrm{~mL} /$ day in developing countries [19].

\subsection{Anthropometry}

Anthropometric measurements were performed as described by Cameron, 2004 [20]. Mothers' body weight and height were measured using an electronic scale (SECA ${ }^{\circledR}-876$, Seca gmbh \& Co., Hamburg, Germany) and a portable stadiometer (SECA ${ }^{\circledR}-217$, Seca gmbh \& Co.), respectively. From both measurements, body mass index (BMI) was calculated and classified according to WHO criteria. Infants' body weight was measured with a pediatric electronic scale $\left(\mathrm{SECA}^{\circledR}-728\right.$, Seca gmbh \& Co.) and length using an infantometer (SECA ${ }^{\circledR}-416$, Seca gmbh \& Co.). Infants' anthropometric indices, i.e., weight for age, length for age and weight for length, were classified according to WHO growth reference Z-scores, using the software Anthro v 3.2.2 (World Health Organization, Geneva, Switzerland) [21].

\subsection{Deuterium Oxide Dilution 'Dose-to-Mother' Technique}

After anthropometric measurements, basal saliva samples from both mother and infant $(5 \mathrm{~mL}$ and $2 \mathrm{~mL}$, respectively) were collected and placed in cryogenic vials, in order to determine the deuterium natural abundance in participant mother-infant pairs. Next, an oral dose of deuterium oxide (deuterium-labeled water) was provided to the mother ( $30 \mathrm{~g}$; Cambridge Isotopes Laboratories, Andover, MA, USA). Deuterium oxide was allowed to mix in the mother's total body water pool, during which it was naturally transferred to the breastfed infant through BM. Mother-infant saliva samples were collected on days 1, 2, 3, 4, 13 and 14 post-dose. All saliva samples were stored at $-20^{\circ} \mathrm{C}$ until their analyses at the Nutrition and Body Composition Laboratory (University of Sonora).

$\mathrm{BM}$ and other liquids consumed by the infant, were calculated according to a water turnover model using deuterium-oxide as a tracer and water as the tracee [17]. Mother's isotopic enrichment followed a single exponential equation:

$$
E_{m(t)}=E_{m(0)} e^{-k m m t}
$$

where $E_{m(t)}$ refers to the isotopic enrichment in the mother's body at time $t(\mathrm{ppm}), E_{m(0)}$ is the enrichment at time zero (ppm), $t$ is the time post-dose saliva sample were taken and $\mathrm{kmm}$ is the water turnover in the mother (1/day).

For each infant, data were fitted as a double exponential based on the fact that infants could receive liquids from BM and non-milk sources. The infant's equation is:

$$
E_{b(t)}=E_{m(0)}\left(\frac{F_{b m}}{V_{b}}\right)\left(\frac{e^{-k_{m m} t}-e^{-\left(F_{b b} / V_{b}\right) t}}{\left(F_{b b} / V_{b}\right)-k_{m m}}\right)
$$


where $E_{b(t)}$ refers to the isotopic enrichment in the infant's body at time $t(\mathrm{ppm}), F_{b m}$ is the water transferred from the mother to the infant through $\mathrm{BM}(\mathrm{kg} /$ day $), V_{b}$ is the infant's total deuterium distribution $(\mathrm{kg})$ and $F_{b b}$ is the total water loss in the infant ( $\mathrm{kg} /$ day).

Parameters fitted are $E_{m(0)}, F_{b m}, K_{m m}$ and $F_{b b} . V_{b}$ is assumed to change in a linear manner with weight $(\mathrm{W}, \mathrm{kg})$ during the experimental period and was related to infant's $\mathrm{W}$ as: $V_{b}=0.84 \mathrm{~W}^{0.82}$ [18].

The deuterium enrichment of the saliva samples was determined by Fourier transform infrared spectrometry (Nicolet iS 10, ThermoScientific, Waltham, MA, USA). BM and non-milk intake were calculated by fitting the deuterium enrichment data to a model of water turnover in the mother and the infant using the Solver function of Microsoft Excel ${ }^{\circledR}$ based on the principles of these equations, proposed by Coward et al. 1982 [17] and validated by the International Atomic Energy Agency (IAEA). Another advantage of using the DOD technique is that at the same time one can measure body composition of the mother (fat-free mass and fat mass) [18].

Infant feeding patterns were classified according to WHO [22] whereby EBF infants receive only BM from their mother and no other liquids or solids with the exception of drops or syrups consisting of vitamins, mineral supplements, or medicines.

\subsection{Breast Milk Sample}

A casual sample of BM was collected, i.e., a small amount of BM at no particular time since the last breastfeeding episode. This method is a recommended biochemical indicator by WHO to assess VA status in lactating women and it is further supported by other studies [23,24].

BM collection was performed by stimulation using a manual breast pump around 11:30 a.m. $( \pm 2 \mathrm{~h})$. After five seconds of milk production, BM samples were collected and then placed in amber glass vials $(5 \mathrm{~mL})$. Subsequently, fresh BM samples were placed on frozen gel packs and stored in an icebox, protected from light, and transported to the Nutrition and Body Composition Laboratory (University of Sonora), where they were stored at $-20{ }^{\circ} \mathrm{C}$ until $\mathrm{BM}$ retinol and carotenoid concentrations were analyzed by HPLC.

In BM, VA is found primarily in the milk fat globules, thus alterations in BM fat content also affect $\mathrm{BM}$ retinol and carotenoid concentrations. Therefore, previous to HPLC analysis, BM samples were thawed, homogenized, and milk fat percentage was calculated using the creamatocrit method described by Lucas et al., 1978 [25].

\subsection{Breast Milk Retinol and Carotenoid Concentrations}

BM VA content was determined using HPLC after an aliquot $(500 \mu \mathrm{L})$ of milk was saponified. Briefly, $750 \mu \mathrm{L}$ ethanol was added and the vial was mixed by vortex $15 \mathrm{~s}$, then $400 \mu \mathrm{L} \mathrm{KOH}: \mathrm{HOH}$ (50:50, w:v) was added and mixed $15 \mathrm{~s}$, and placed in a water bath at $45^{\circ} \mathrm{C}$ for $60 \mathrm{~min}$, mixing the vials every $15 \mathrm{~min}$ [10]. Later the sample was extracted twice with $1 \mathrm{~mL}$ hexanes, mixed for $30 \mathrm{~s}$, and centrifuged $10 \mathrm{~min}$ at $4{ }^{\circ} \mathrm{C}$ and $3000 \mathrm{rpm}$. The upper hexane layer was collected and placed in a test tube for subsequent evaporation under a gentle stream of nitrogen. The residue was reconstituted in $100 \mu \mathrm{L}$ ethanol and injected onto the HPLC system using a C30 YMC S-3-Carotenoid $(3 \mu \mathrm{m}, 150 \times 4.6 \mathrm{~mm})$ column and the method described by Yeum et al., 1996 [26]. The system was an HPLC Agilent-1220 equipped with a photodiode array detector (Infinity 1260, Agilent Technologies, Santa Clara, SA, USA).

For quality control we used echinenone as an internal standard. HPLC-purified standards were used to calculate retinol, lutein, zeaxanthin, $\beta$-cryptoxanthin, $\alpha$-carotene, all-trans- $\beta$-carotene, and lycopene concentrations by using an external calibration curve obtained from standards (Sigma-Aldrich, St. Louis, MO, USA). The inter-run variation was $9 \%$ for retinol and $15 \%$ for total carotenoids (based on the analysis of pooled BM sample).

$\mathrm{BM}$ retinol and provitamin A carotenoid concentrations were expressed by volume ( $\mu \mathrm{mol} / \mathrm{L}$ and $\mathrm{nmol} / \mathrm{L}$, respectively), and retinol was also expressed per gram of milk fat ( $\mu \mathrm{g}$ retinol/g milk fat). According to $\mathrm{WHO}$, in lactating women VAD is defined when $\mathrm{BM}$ retinol concentration is $<1.05 \mu \mathrm{mol} / \mathrm{L}$ or $\leq 8 \mu \mathrm{g} \mathrm{retinol/g}$ milk fat [1]. 


\subsection{Adequacy of Infants' Vitamin A Intake}

To assess whether consumption of VA in participant infants was adequate, we considered the volume of milk that was consumed per day and the BM retinol concentration by volume. Additionally, we considered the contribution of BM provitamin A carotenoids to the infant's VA dietary intake using the conversion factors recommended by the Institute of Medicine-Food and Nutrition Board (i.e., $12 \mu \mathrm{g} \beta$-carotene and $24 \mu \mathrm{g} \beta$-cryptoxanthin and $\alpha$-carotene per $1 \mu \mathrm{g}$ of retinol). Breastfed infant's VA intake was then compared with the recommended dietary adequate VA intake (i.e., 400 retinol activity equivalents (RAE)/day) [27].

\subsection{Statistical Analysis}

Descriptive statistics were generated. Values in the text are means \pm standard deviations. Analysis included proportions for categorical variables and measures of central tendency for continuous variables. Differences between groups were examined by $\chi^{2}$ test, Student $t$-test or ANCOVA for parametric variables, and Mann-Whitney $U$ or Wilcoxon test for nonparametric variables. For continuous variables, tests were performed assuming a 2-tailed analysis and differences were considered significant at $p<0.05$. Correlations between variables of interest were assessed by Pearson's correlation coefficient. Data analysis was performed using the NCSS statistical software (NCSS 2016 ${ }^{\circledR}$, Kaysville, UT, USA, v 11.0.6).

\section{Results}

Fifty-nine lactating women were enrolled, but 3 were excluded from the analysis because BM intake by the infants was $<250 \mathrm{~mL} /$ day. Analyzed data include the 56 lactating women-infant pairs that met the criteria for participation (26 and 30 from the urban and agricultural areas, respectively). Table 1 shows the general characteristics of the mother-infant pairs.

When assessing lactating women's BMI individually, 5.4\% $(n=3), 41 \%(n=23)$ and $53.6 \%(n=30)$ were classified as low weight, normal, and overweight, respectively, and no difference was observed between areas $(p=0.16)$. There was a positive association between fat mass percentage and BMI in participant lactating mothers $(p<0.01)$, and 60.7\% $(n=34)$ of them showed high levels of body fat, when contrasted with the reference values for non-pregnant and lactating women [28].

Even when the average for all nutritional indicators of participant infants were normal, based on the individual analysis of the $\mathrm{Z}$ scores of anthropometric indicators, 6 infants were below $-2 \mathrm{Z}$ scores of weight/length ( 2 and 4 from the urban and agricultural areas, respectively) and 1 was below $-2 \mathrm{Z}$ scores of height/age (urban).

Table 1. General characteristics of participant mothers and infants.

\begin{tabular}{ccccc}
\hline & Total $(\boldsymbol{n}=\mathbf{5 6})$ & Urban Area $(\boldsymbol{n}=\mathbf{2 6})$ & Agricultural Area $(\boldsymbol{n}=\mathbf{3 0})$ & $p$ \\
\cline { 2 - 5 } & \multicolumn{4}{c}{ Mean \pm Standard Deviation } \\
Mothers & & & \\
Age (Years) & $25 \pm 7$ & $27.8 \pm 6$ & $22.4 \pm 7$ & 0.003 \\
Weight (kg) & $62 \pm 12.8$ & $63 \pm 11.5$ & $70 \pm 14$ & 0.49 \\
Height (cm) & $157 \pm 8.6$ & $161 \pm 6.3$ & 0.000 \\
BMI & $25 \pm 4.8$ & $24.5 \pm 4$ & $26 \pm 5$ & 0.26 \\
\hline Infants & $3 \pm 2$ & $3.6 \pm 1.5$ & $3.1 \pm 1.6$ & 0.24 \\
Age (month) & $6 \pm 1$ & $6.4 \pm 0.9$ & $6.1 \pm 1.4$ & 0.30 \\
Weight (kg) & $61 \pm 4.5$ & $61.1 \pm 3.7$ & $60 \pm 5$ & 0.51 \\
Length (cm) & $0.1 \pm 1.2$ & $0.1 \pm 1.0$ & $0 \pm 1.3$ & 0.65 \\
Weight for age (Z score) & $-0.1 \pm 1.5$ & $-0.2 \pm 1.3$ & $0 \pm 1.6$ & 0.51 \\
Length for age (Z score) & $0.3 \pm 1.4$ & $0.5 \pm 1.4$ & $0.2 \pm 1.4$ & \\
Weight for length (Z score) & & & & \\
\hline
\end{tabular}

$\mathrm{BM}$ retinol and carotenoid concentrations are shown in Table 2. Mean BM retinol concentration was $1.1 \mu \mathrm{mol} / \mathrm{L}$, and when comparing to the cut-off point for deficiency (i.e., BM retinol $<1.05 \mu \mathrm{mol} / \mathrm{L}$ ), 
$57 \%(n=32)$ of participant women had VAD. Even though BM retinol concentration (by volume) was significantly lower in the agricultural area $(p=0.028)$, the prevalence of VAD was similar in both areas, $60 \%(n=18)$ and $54 \%(n=14)$, for the urban and agricultural areas, respectively $(p=0.64)$.

Table 2. Concentration of retinol and carotenoids found in breast milk of participant lactating mothers.

\begin{tabular}{|c|c|c|c|c|}
\hline & Total & Urban Area $(n=26)$ & Agricultural Area $(n=30)$ & $p$ \\
\hline Retinol $\mu \mathrm{mol} / \mathrm{L}$ & $1.1 \pm 0.6$ & $1.3 \pm 0.69$ & $0.92 \pm 0.51$ & 0.028 \\
\hline Lutein (nmol/L) & $30.7 \pm 21.2$ & $36 \pm 21.6$ & $26 \pm 20$ & 0.083 \\
\hline Zeaxanthin (nmol/L) & $7.7 \pm 4.4$ & $8.9 \pm 5.2$ & $6.8 \pm 3.4$ & 0.073 \\
\hline$\beta$-cryptoxanthin (nmol/L) & $2.5 \pm 3.2$ & $4 \pm 4.2$ & $1.2 \pm 0.7$ & 0.001 \\
\hline Lycopene (nmol/L) & $1.1 \pm 0.9$ & $1.5 \pm 1.2$ & $0.73 \pm 0.60$ & 0.002 \\
\hline Provitamin A carotenoids (nmol/L) & $65.7 \pm 84.5$ & $105 \pm 110$ & $31.2 \pm 23.2$ & 0.001 \\
\hline Total BM carotenoids (nmol/L) & $105 \pm 104$ & $152 \pm 133$ & $64.8 \pm 40.1$ & 0.001 \\
\hline
\end{tabular}

Fat is an important factor to account for when assessing retinol and carotenoid concentrations in BM. In our study population, BM fat concentration was $23 \pm 14 \mathrm{~g} / \mathrm{L}$ and no difference was observed between areas $(p=0.43)$. Calculating the VA concentration ( $\mu$ g retinol) per gram of milk fat as an alternative indicator of VA status for lactating women, VAD was identified in only $16 \%(n=9)(p=0.15)$. We analyzed the association between body composition categorized by BMI (<18: thinnest; 18-24.9: normal and $\geq 25$ : overweight) and BM retinol concentration (by volume and per gram of milk fat), and there was no statistical difference ( $p>0.05$ for both variables) (Table 3). Although, when analyzing the relationship of stunting in early life of participant mothers on their VA status, stunted women $(n=14)$ tended to have lower BM retinol concentration $(p=0.058)$ than those with normal height.

Table 3. Vitamin A concentration (by volume or per gram of milk fat) and fat mass percentage categorized by body mass index (BMI) (<18: thinnest; 18-24.9: normal and $\geq 25$ : overweight).

\begin{tabular}{cccc}
\hline BMI & \% Fat Mass & VA $\mu$ mol/L & VA $\mu$ g/g Fat \\
\hline \multicolumn{3}{c}{ Mean \pm Standard Deviation } \\
\hline$<18(n=3)$ & $24 \pm 0.4^{\mathrm{a}}$ & $0.74 \pm 0.2$ & $10 \pm 1.7$ \\
$18-24.9(n=23)$ & $29 \pm 8.6^{\mathrm{a}}$ & $1.06 \pm 0.6$ & $16 \pm 8$ \\
$\geq 25(n=30)$ & $38 \pm 5.4^{\mathrm{b}}$ & $1.15 \pm 0.6$ & $16 \pm 9$ \\
$p$ & 0.000 & 0.533 & 0.491 \\
\hline \multicolumn{4}{c}{${ }^{\mathrm{a}, \mathrm{b}}$ Different letter in column means statistical difference. }
\end{tabular}

The major provitamin A carotenoid found in BM was $\beta$-carotene (95\%), followed by $\beta$-cryptoxanthin $(4 \%)$ and $\alpha$-carotene $(1 \%)$. When considering total carotenoids in BM, $\beta$-carotene was still the most abundant (50\%), followed by lutein (34\%), zeaxanthin $(10 \%)$, $\beta$-cryptoxanthin $(4 \%)$, $\alpha$-carotene $(1 \%)$, and lycopene (1\%). There was a positive and significant association between BM retinol and provitamin A carotenoid concentrations: $\alpha$-carotene $(r=0.61 ; p<0.001)$, $\beta$-carotene $(r=0.58$; $p<0.001)$ and $\beta$-cryptoxanthin $(r=0.43 ; p<0.001)$.

Table 4 shows the BM intake by the infants as well as their VA dietary adequacy, either from preformed VA (retinol) or provitamin A carotenoids in addition to preformed VA. BM intake tended to be lower in infants from the urban area $(p=0.067)$. Given the precision of the dose-to-mother method we were able to identify EBF (non-BM fluid intake $71 \pm 56 \mathrm{~g} /$ day) in $62.5 \%(n=35)$ and PBF (non-BM fluid intake $427 \pm 176 \mathrm{~g}$ /day) in $37.5 \%(n=21)$ of participant lactating mothers. The BM intake tended to be higher for EBF infants $(777 \mathrm{~mL})$ vs those PBF $(599 \mathrm{~mL})(p=0.05)$, also there was no statistical difference in BM retinol concentration between EBF and PBF (either by volume or per gram of milk fat). Additionally, by comparing the dose-to-mother results with the mother's declaration of type of breastfeeding, it was possible to observe that $70 \%(n=39)$ accurately described if they were EBF or PBF. 
Table 4. Breast milk intake and adequacy of intake of vitamin A (VA) in participating infants.

\begin{tabular}{|c|c|c|c|c|}
\hline & Total $(n=59)$ & Urban Area $(n=26)$ & Agricultural Area $(n=30)$ & $p$ \\
\hline Breast milk intake (mL) & $758 \pm 185$ & $710 \pm 169$ & $800 \pm 190$ & 0.067 \\
\hline Adjusted retinol intake per $\mathrm{g}$ of $\mathrm{BM}$ fat $(\mu \mathrm{g} / \text { day })^{1}$ & $229 \pm 129$ & $259 \pm 23 *$ & $202 \pm 21 *$ & 0.072 \\
\hline VA $^{2}$ intake (RAE/day) & $231 \pm 130$ & $268 \pm 157$ & $198 \pm 93.6$ & 0.043 \\
\hline Mean Adequacy of VA intake (\%) & $57 \pm 32$ & $66 \pm 39$ & $49 \pm 23$ & 0.054 \\
\hline
\end{tabular}

${ }^{1} \mathrm{BM}$ retinol intake for agricultural and urban areas was adjusted using BM fat concentration [25] as a covariable.

* Values are presented as adjusted means and standard error. ${ }^{2}$ VA: includes retinol and provitamin A carotenoids

( $\alpha$ - and $\beta$-carotene and $\beta$-cryptoxanthin).

According to the intake of BM by the infants and the amount of retinol and provitamin $\mathrm{A}$ carotenoids in BM, we observed that infants from the agricultural area tended to have a lower VA adequacy compared with those from the urban area $(p=0.054)$. On average, infants only consumed 229 out of $400 \mathrm{RAE} /$ day (either quantified by volume or per gram of fat), which is the adequate intake for this age group. Taking into account the above, failure to meet the daily VA recommended intake was observed in $77 \%(n=20)$ and $97 \%(n=29)$ of breastfed infants from the urban and agricultural areas, respectively. Considering the mother's nutritional status, infants from stunted mothers met only $41 \%$ of their daily recommended intake vs. $63 \%$ on infants of women with normal height $(p=0.029)$.

After considering provitamin A carotenoids as part of the daily dietary VA intake of participant breastfed infants, a small but significant increase was observed (231 RAE; $p<0.001$ ); and this led to a reduction in VA inadequacy from $88 \%$ to $86 \%$. As expected, we found a positive and significant association between total VA intake and the amount of fat in BM $(r=0.43 ; p=0.001)$.

\section{Discussion}

This study combined two methodologies (i.e., dose-to-mother DOD and BM retinol concentration), which allowed us to assess the VA status of the mother, as well as the VA intake of the breastfed infant. An advantage of our study design lies in the reduction of experimental bias, because the DOD technique quantifies an accurate amount of BM consumed by the breastfed infant and does not rely on weighing the infant before and after a feed. Additionally, the dose-to-mother method discriminates between EBF and PBF. As seen in our results, only $70 \%$ of participant women accurately self-reported $\mathrm{EBF}$ or $\mathrm{PBF}$, this may represent bias when interpreting results from studies that rely only on self-report breastfeeding practices.

The theoretical consumption of milk used by other authors is between $600 \mathrm{~mL} /$ day [29] and $800 \mathrm{~mL} /$ day [30]. Furthermore, the US Institute of Medicine established the daily adequate intake of VA for breastfed infants based on a theoretical BM consumption of $780 \mathrm{~mL} /$ day. The BM intake by enrolled infants was within the range mentioned above and consistent with data published by other authors who have used the 'dose-to-mother' technique, $759 \pm 142 \mathrm{~mL} /$ day [31] and $637 \pm 247 \mathrm{~mL} /$ day [32], to quantify consumption of BM by the infant. On the other hand, we have the limitation of the collection of a casual sample of BM which may not reflect the diurnal variations in BM fat $[33,34]$ and this may affect the concentration of VA. Nonetheless, this is a recommended biochemical indicator by WHO and has been supported by other studies [23,24]. Furthermore, we standardized the time of collection to be at 11:30 a.m. $\pm 2 \mathrm{~h}$ to reduce variation.

According to $\mathrm{WHO}$, the percentage of VAD observed among participant lactating women using BM retinol as an indicator, represents a problem of public health concern [1]. Fifty seven percent of women showed low BM retinol $(\mu \mathrm{mol} / \mathrm{L})$, which drops to $16 \%$ when corrected for BM fat, confirming other findings that list Mexico as a country where VAD is a public health problem in different population groups [1]. Regardless of adjustments, VA intake of these infants is still below the recommended dietary intake [27]. Correction for fat proved to be important in this study, but logistically it is often cumbersome to determine in population surveys. This is especially true when the creamatocrit and volume aliquots cannot be performed on fresh milk, which then requires 
re-homogenization before analysis in the laboratory [10]. Additionally, at a national level often rural areas are most affected by VAD [5], which was also observed in our results, where the women of the agricultural area had significantly lower concentrations of BM retinol, when compared with those of the urban area $(p<0.05)$. Recent findings in Thailand show that BM retinol in hindmilk, which typically has higher fat content, is strongly associated with total body VA stores in lactating women (Tanumihardjo, personal communication) [35]. Even though hindmilk was not specifically collected in our study, the prevalence of low BM retinol is high and we can infer that participant lactating women's VA stores may be depleted.

All infants are born with low stores of VA and BM is the infant's main source of VA to meet requirements and build liver stores [36], but in areas where VAD is common, concentrations of BM retinol are observed to be $1 \mu \mathrm{mol} / \mathrm{L}$ [3], similar to our study. This amount only meets the metabolic needs of the baby and does not allow accumulation of liver stores of this nutrient [4]. Furthermore, our results suggest that the mother's nutritional status (i.e., malnutrition in early life) was a limiting factor for VA adequacy of the breastfed infant $(p=0.029)$, and thus should be a variable to consider when assessing the VA status of lactating women.

A trend towards increased consumption of BM for infants from the agricultural area occurred $(p=0.067)$, perhaps as a compensatory mechanism to receive a greater amount of nutrients, given that no difference was found between areas when using BM retinol concentration by gram of fat $(p=0.438)$. There is an assumption that BM from well-nourished mothers in developing countries has an average of $38 \mathrm{~g} / \mathrm{L}$ of fat, when $24 \mathrm{~h} \mathrm{BM}$ collection is performed [37,38]. We compared our results with a calculated estimate of vitamin A intake assuming that BM fat was a constant of $38 \mathrm{~g} / \mathrm{L}$ for all participant women, and the result (i.e., $404 \mu \mathrm{g}$ VA/day) almost doubles our quantified VA intake per gram of BM fat $(p=0.000 ; 95 \mathrm{CI} 314-504 \mu \mathrm{g} /$ day). Additionally, inadequacy of VA intake was reduced to $50 \% \mathrm{vs} .88 \%$ found in our study. Hence, this reiterates the importance of the dose-to-mother method, and when possible, individual determinations of BM fat concentration in the field to accurately estimate BM volume and adequacy of fat-soluble micronutrient intakes by the breastfed child. Although BM fat analysis is logistically difficult in population studies, using a fixed concentration of BM fat can mask nutritional deficiencies, especially in developing countries where mothers may not have an adequate nutritional status.

When assessing the VA adequacy of breastfed infants, the Institute of Medicine established the daily adequate intake based on the consumption of $780 \mathrm{~mL} \mathrm{BM} /$ day by the infant. We compared our results (i.e., quantified BM intake) with a calculated estimate of VA intake assuming only $780 \mathrm{~mL}$ $\mathrm{BM} /$ day and no statistical difference was found $(p>0.05)$, perhaps because the BM intake of our infants was close to the Institute of Medicine's assumption. Additionally, the Institute of Medicine does not consider the contribution of provitamin A carotenoids to meet the infants VA requirement, because the bioconversion of carotenoids from milk in infants is not known [27]. Nonetheless, we explored the impact of including the carotenoid intake from BM on the VA dietary adequacy of the infants and it showed a significant increase in VA intake $(p<0.001)$. We also used a high bioconversion factor, i.e., $12 \mu \mathrm{g} \beta$-carotene equivalents to $1 \mu \mathrm{g}$ retinol, which likely underrepresents the actual bioconversion rate in infants with VAD.

Total carotenoid concentration observed in this study $(105 \mathrm{nmol} / \mathrm{L})$ considerably differs from a previous study on carotenoid content in BM of Mexican well-nourished lactating women $(223 \mathrm{nmol} / \mathrm{L})$ but is similar to that observed in women from the US (114 nmol/L) [39]. This can be explained by the similarities in dietary habits of the northwest region of Mexico and the US. The carotenoid profile found in this study differs from what Canfield et al. [39], described for Mexican lactating women where $\beta$-cryptoxanthin was the most abundant carotenoid, but is similar to a more recent report by Lipkie et al. 2015 [40] in Mexican lactating women where $\beta$-carotene was the most abundant.

An additional advantage of the 'dose-to-mother' technique is the simultaneous assessment of the lactating mother's body composition. At present, cut-off points to define excess or limited body fat percentage in lactating women are not available, but when considering body fat combined with 
a BMI $\geq 25$ more than $50 \%$ of participant women were overweight or obese. Nevertheless, the physiological rise in fat stores during pregnancy supports lactating women in BM production given that EBF women require over $640 \mathrm{kcal}$ in addition to their pre-pregnancy energy requirement [41]. Even when there are not enough data to estimate the amount of body fat lactating women must have during lactation, our study showed that their fat stores were sufficient to sustain an average of $758 \mathrm{~mL} /$ day of BM production. On the other hand, we found that $25 \%(n=14)$ of the women had low height $(<150 \mathrm{~cm})$ [42], which is a sign of malnutrition in early life, and these women tended to have lower BM retinol concentrations when compared to those of normal stature $(p=0.058)$.

By combining the 'dose-to-mother' method with the determination of BM retinol, it was possible to demonstrate VAD in the population and quantify the amount of VA that the infants received while breast-feeding. Although serum retinol concentrations were not determined directly in the infants, which is a limitation of this study, BM retinol concentrations combined with BM volume suggested low VA intakes.

\section{Conclusions}

BM is the only source of food for EBF infants, and because of the high prevalence of VAD among Mexican lactating women, strategies to reduce VAD in the population must target both children under 5 years as well as lactating women, in order to increase the chances of improving VA status in both groups. The use of nuclear techniques (stable isotopes) in vulnerable populations, particularly in lactating women and infants, such as the dose-to-mother DOD and stable retinol isotope dilution, could improve status assessment of VA and provide a better and more accurate estimate of VA dietary requirements for breastfed infants.

Acknowledgments: This research was funded by Programa para el Desarrollo Profesional Docente (PRODEP) and internal finance by University of Sonora Division of Biological Sciences and Health (USO313001242). A.T.L.M. received a fellowship from CONACyT. Special thanks to Roxana Leticia Castro González and Maribel Ramírez Torres for their collaboration in careful data collection during field work.

Author Contributions: V.L.T. and M.E.V. conceived and designed the experiments; V.L.T., A.T.L.-M. and O.T.-O. performed the experiments; V.L.T. and M.E.V. analyzed the data; V.L.T. and H.A.-G. contributed reagents/materials/analysis tools; V.L.T. and S.A.T. wrote the paper.

Conflicts of Interest: The authors declare no conflict of interest. The founding sponsors had no role in the design of the study; in the collection, analyses, or interpretation of data; in the writing of the manuscript, and in the decision to publish the results.

\section{References}

1. World Health Organization. Global prevalence of vitamin A deficiency in populations at risk 1995-2005. In WHO Global Database on Vitamin A Deficiency; World Health Organization: Geneva, Switzerland, 2009.

2. Duarte, M.E. Retinol en Suero y Leche Materna y su Asociación con la Ingesta Dietaria de Vitamina A en Mujeres en Periodo de Lactancia que Acuden al Hospital Integral de la Mujer del Estado de Sonora (HIMES). Master's Thesis, Universidad de Sonora, Hermosillo, Mexico, 2013.

3. Wallingford, J.; Underwood, B.A. Vitamin A status needed to maintain vitamin A concentration in nonhepatic tissues of the pregnant rat. J. Nutr. 1987, 117, 1410-1415. [PubMed]

4. Underwood, B.A. Maternal vitamin A status and its importance in infancy and early childhood. Am. J. Clin. Nutr. 1994, 59, 517-524.

5. Villalpando, S.; de la Cruz, V.; Shamah, T.; Rebollar, R.; Contreras-Manzano, A. Nutritional status of iron, vitamin B12, folate, retinol and anemia in children 1 to 11 years old. Results of the Ensanut 2012. Salud. Publ. Mex. 2015, 57, 372-384. [CrossRef]

6. Robles, A.; Astiazaran, H.; Dávalos, N.; Quihui, L.; Cabrera, P.; Valencia, M. Efecto de la suplementación masiva de vitamina A en niños de 6 a 36 meses de edad. Salud. Publ. Mex. 1998, 40, 309-315. [CrossRef] 
7. Lopez-Teros, V.; Quihui-Cota, L.; Méndez-Estrada, R.O.; Grijalva-Haro, M.I.; Esparza-Romero, J.; Valencia, M.E.; Green, M.H.; Tang, G.; Pacheco-Moreno, B.I.; Tortoledo-Ortiz, O.; et al. Vitamin A fortified milk increases total body vitamin A stores in Mexican preschoolers. J. Nutr. 2013, 143, 221-226. [CrossRef] [PubMed]

8. Stoltzfus, R.J.; Underwood, B.A. Breast-milk vitamin A as an indicator of the vitamin A status of women and infants. Bull. World Health Organ. 1995, 73, 703-711. [PubMed]

9. De Pee, S.; Dary, O. Biochemical indicators of vitamin A deficiency: Serum retinol and serum retinol binding protein. J. Nutr. 2002, 132, 2895S-2901S. [PubMed]

10. Tanumihardjo, S.A.; Penniston, K.L. Simplified methodology to determine breast milk retinol concentrations. J. Lipid Res. 2002, 43, 350-355. [PubMed]

11. Surles, R.L.; Li, J.; Tanumihardjo, S.A. The modified-relative-dose-response value in serum and milk are positively correlated over time in lactating sows with adequate vitamin A status. J. Nutr. 2006, 136, 939-945. [PubMed]

12. Dancheck, B.; Nussenblat, V.; Ricks, M.O.; Kumwenda, N.; Neville, M.C.; Moncrief, D.T.; Taha, T.E.; Semba, R.D. Breast milk retinol concentrations are not associated with systemic inflammation among breast-feeding women in Malawi. J. Nutr. 2005, 135, 223-226. [PubMed]

13. Ross, A.C.; Pasatiempo, A.M.; Green, M.H. Chylomicron margination, lipolysis, and vitamin A uptake in the lactating rat mammary gland: Implications for milk retinoid content. Exp. Biol. Med. 2004, 229, 46-55.

14. Raiten, D.J.; Sakr Ashour, F.A.; Ross, A.C.; Meydani, S.N.; Dawson, H.D.; Stephensen, C.B.; Brabin, B.J.; Suchdev, P.; van Ommen, B. INSPIRE Consultative Group. Inflammation and nutritional science for programs/policies and interpretation of research evidence (INSPIRE). J. Nutr. 2015, 145, 1039S-1108S. [CrossRef] [PubMed]

15. Food and Agriculture Organization; World Health Organization; United Nations University. Energy and Protein Requirements: Report of the Joint FAO/WHO Ad Hoc RI Expert Committee; WHO Technical Report Series 724; WHO: Geneva, Switzerland, 1985.

16. Agne-Djingo, A.; Idohou-Dossou, N.; Kwadjode, K.; Tanumihardjo, S.; Wade, S. High prevalence of vitamin A deficiency is detected by the modified relative dose-response test in six-month-old Senegalese breast-fed infants. J. Nutr. 2012, 142, 1991-1996. [CrossRef] [PubMed]

17. Coward, W.A.; Cole, T.J.; Sawyer, M.B.; Prentice, A.M. Breast-milk intake measurement in mixed-fed infants by administration of deuterium oxide to their mothers. Hum. Nutr. Clin. Nutr. 1982, 36C, 141-148.

18. International Atomic Energy Agency. Human Health Series No. 7. In Stable Isotope Technique to Assess Intake of Human Milk in Breastfed Infants, 1st ed.; IAEA: Vienna, Austria, 2010.

19. Butte, N.F.; King, J.C. Energy requirements during pregnancy and lactation. Public Health Nutr. 2005, 8, 1010-1027. [CrossRef] [PubMed]

20. Cameron, N. Measuring Growth. In Methods in Human Growth Research; Hauspie, R., Cameron, N., Molinary, L., Eds.; Cambridge University Press: Cambridge, UK, 2004; pp. 68-107.

21. World Health Organization. Child Growth Standards: Length/Height-for-Age, Weight-for-Age, Weight-for-Length, Weight-for-Height and Body Mass Index-for-Age: Methods and Development; World Health Organization: Geneva, Switzerland, 2006.

22. World Health Organization. Global Strategy for Infant and Young Child Feeding: The Optimal Duration of Exclusive Breast-Feeding; World Health Organization: Geneva, Switzerland, 2001.

23. World Health Organization; United Nations International Children's Fund. Indicators for Assessing Vitamin A Deficiency and Their Application in Monitoring and Evaluating Intervention Programmes; WHO: Geneva, Switzerland, 1996.

24. Rice, A.L.; Stoltzfus, R.J.; de Francisco, A.; Kjolhede, C.L. Evaluation of serum retinol, the modified-relativedose-response ratio, and breast-milk vitamin A as indicators of response to postpartum maternal vitamin $\mathrm{A}$ supplementation. Am. J. Clin. Nutr. 2000, 71, 799-806. [PubMed]

25. Lucas, A.; Gibbs, J.A.H.; Lyster, R.L.J.; Baum, J.D. Creamatocrit: Simple clinical technique for estimating fat concentration and energy value of human milk. Br. Med. J. 1978, 1, 1018-1020. [CrossRef] [PubMed]

26. Yeum, K.J.; Booth, S.; Sadowski, J.; Liu, C.; Tang, G.; Krinski, N.; Russell, R. Human plasma carotenoid response to the ingestion of controlled diets high in fruits and vegetables. Am. J. Clin. Nutr. 1996, 64, 594-602. [PubMed] 
27. Institute of Medicine-Food and Nutrition Board. Dietary Reference Intakes for Vitamin A, Vitamin K, Arsenic, Boron, Chromium, Copper, Iodine, Iron, Manganese, Molybdenum, Nickel, Silicon, Vanadium and Zinc: A Report of the Panel on Micronutrients, Subcommittees on Upper Reference Levels of Nutrients and of Interpretation and Uses of Dietary Reference Intakes and the Standing Committee on the Scientific evaluation of Dietary Reference Intakes; National Academy Press: Washington, DC, USA, 2001.

28. Gallagher, D.; Heymsfield, S.B.; Heo, M.; Jebb, S.A.; Murgatroyd, P.R.; Sakamoto, Y. Healthy percentage body fat ranges: An approach for developing guidelines based on body mass index. Am. J. Clin. Nutr. 2000, 72, 694-701. [PubMed]

29. Vargas, A.; Vallejo, M. Residuos de Insecticidas Organoclorados en Leche Humana y de Vaca en Colombia. Bol. Sanit. Panam. 1990, 108, 220-228.

30. Waliszewski, S.M.; Pardio, V.T.; Chantiri, J.N.; Infanzon, R.M.; Rivera, J. Organochlorine pesticide residues in adipose tissue of Mexicans. Sci. Total Environ. 1996, 181, 125-131. [CrossRef]

31. Dusdieker, L.; Hemingway, D.; Stumbo, P. Is milk production impaired by dieting during lactation? Am. J. Clin. Nutr. 1994, 59, 833-840. [CrossRef] [PubMed]

32. Nafeesa, N.; Rakshanda, B.; Zahid, L.; Les, B. Maternal body composition and its relationship to infant milk intake in rural Pakistan. Food Nutr. Sci. 2011, 2, 932-937.

33. Saarela, T.; Kokkonen, J.; Koivisto, M. Macronutrient and energy contents of human milk fractions during the first six months of lactation. Acta Paediatr. 2005, 94, 1176-1181. [CrossRef] [PubMed]

34. Kent, J.C.; Mitoulas, L.R.; Cregan, M.D.; Ramsay, D.T.; Doherty, D.A.; Hartmann, P.E. Volume and frequency of breastfeedings and fat content of breast milk throughout the day. Pediatrics 2006, 117, e387-e395. [CrossRef] [PubMed]

35. Tanumihardjo, S.A.; (Department of Nutritional Sciences, University of Wisconsin-Madison, Madison, WI, USA). Personal communication, 2016.

36. Haskell, M.J.; Brown, K.H. Maternal vitamin A nutriture and vitamin A content of human milk. J. Mammary Gland Biol. Neoplas. 1999, 4, 243-257. [CrossRef]

37. Pan American Health Orgaization; World Health Organization. Guiding Principles for Complementary Feeding of the Breastfed Child; Division of Health Promotion and Protection/Food and Nutrition Program: Washington, DC, USA, 2003.

38. Dewey, K.G.; Brown, K.H. Update on technical issues concerning complementary feeding of young children in developing countries and implications for intervention programs. Food Nutr. Bull. 2003, 24, 5-28. [CrossRef] [PubMed]

39. Canfield, L.M.; Clandinin, M.T.; Davies, D.P.; Fernandez, M.C.; Jackson, J.; Hawkes, J.; Goldman, W.J.; Pramuk, K.; Reyes, H.; Sablan, B.; et al. Multinational study of major breast milk carotenoids of healthy mothers. Eur. J. Nutr. 2003, 42, 133-141. [PubMed]

40. Lipkie, T.E.; Morrow, A.L.; Jouni, Z.E.; McMahon, R.J.; Ferruzzi, M.G. Longitudinal survey of carotenoids in human milk from urban cohorts in China, Mexico, and the USA. PLoS ONE 2015, 10, e0127729. [CrossRef] [PubMed]

41. NRC (National Research Council). Recommended Dietary Allowances, 10th ed.; Report of the Subcommittee on the Tenth Edition of the RDAs, Food and Nutrition Board, Commission on Life Sciences; National Academy Press: Washington, DC, USA, 1989; p. 284.

42. Norma Oficial Mexicana NOM-008-SSA3-2010, Para el Tratamiento Integral del Sobrepeso y la Obesidad. Official Mexican Norm for the Integral Treatment of Overweight and Obesity. Available online: http:/ /www.dof.gob.mx/ (accessed on 31 October 2016).

(c) 2017 by the authors. Licensee MDPI, Basel, Switzerland. This article is an open access article distributed under the terms and conditions of the Creative Commons Attribution (CC BY) license (http://creativecommons.org/licenses/by/4.0/). 